\title{
INTRODUCTION TO THE SPECIAL ISSUE ON ENABLING TECHNOLOGIES FOR COLLABORATIONS
}

\section{Dear SCPE readers,}

Collaboration is an important aspect in almost all fields of human life, and today the need for supporting collaboration is increased by the fact that we are always connected by means of different kinds of devices. In particular in the enterprise world, this need has emerged and satisfying it can lead to relevant benefits for companies. In the last years, enabling technologies have evolved to meet new and challenging requirements. The aim of this special issue is to provide a selection of the state of the art, emerging trends, new technologies and best practices in the field of technologies that enable collaboration. The idea was born at the 2014 IEEE WETICE Conference on Enabling Technology: Infrastructure for Collaborative Enterprises, but the call was open to any submission.

This special issue features three articles that concern technologies that enable collaboration.

The first one, "Engineering And Implementing Software Architectural Patterns Based On Feedback Loops" by Dhaminda B. Abeywickrama, Nicklas Hoch and Franco Zambonelli, focuses on collaboration in decentralized system of autonomous service components. The paper proposes an Eclipse plug-in called SimSOTA, which supports the design and the implementation of self-adaptive systems. Different phases of the development are covered in a model-driven fashion based on self-adaptive architectural patterns.

The second one, "Simulation Data Sharing to Foster Teamwork Collaboration" by Claudio Gargiulo, Delfina Malandrino, Donato Pirozzi and Vittorio Scarano, addresses the collaboration among engineers involved in Computational Fluid Dynamics (CFD) simulations. A Web-based system is presented, called Floasys, which was developed starting from the experience in a big automotive company.

The third paper, "Investigation On The Optimal Properties Of Semi Active Control Devices With Continuous Control For Equipment Isolation" by Michela Basili and Maurizio De Angelis, faces the collaboration among devices, composing a single equipment. A control algorithm, derived from the Lyapunov method and adapted to the addressed problem is presented, which is able to achieve the optimal isolation properties of semi active variable stiffness devices with continuous control across the whole frequency spectrum.

It is interesting to remark that all the accepted papers present a strong connection with real requirements, but meet them by solid models or theory. We also hope that these papers can solicit new research directions in the field.

We would like to thank the editorial board of SCPE for the chance of arranging this special issue, and all the reviewers for their hard work.

Giacomo Cabri, Federico Bergenti, Marco Aiello, Sumitra Reddy and Ramana Reddy 